\title{
RESPOSTA DA LARANJEIRA-PÊRA À ADUBAC̄̃̃O COM NITROGÊNIO, FÓSFORO E POTÁSSIO EM UM LATOSSOLO AMARELO DOS TABULEIROS COSTEIROS ${ }^{1}$
}

\author{
LAFAYETTE FRANCO SOBRAL ${ }^{2}$, LUIZ FRANCISCO DA SILVA SOUZA ${ }^{3}$, \\ ANTÔNIA FONSECA DE JESUS MAGALHÃES ${ }^{3}$, JOSÉ UNALDO BARBOSA SILVA ${ }^{4}$ \\ e MARIA DE LOURDES SILVA LEAL ${ }^{5}$
}

\begin{abstract}
RESUMO - Com o objetivo de estudar os efeitos de N, P e K na produção e qualidade de laranjas-pêra (Citrus sinensis (L.) Osbeck), foi instalado um experimento fatorial, em um Latossolo Amarelo dos Tabuleiros Costeiros. As doses de N, P e K foram, respectivamente, de 0, 150 e $300 \mathrm{~kg} \mathrm{ha}^{-1}$ de N na forma de uréia, 0, 26 e $52 \mathrm{~kg} \mathrm{ha}^{-1}$ de P na forma de superfosfato triplo, e de 0,96 e $192 \mathrm{~kg} \mathrm{ha}^{-1}$ de K na forma de cloreto de $\mathrm{K}$. A resposta ao $\mathrm{N}$ foi linear e positiva em um dos quatro anos observados. $\mathrm{O}$ nutriente diminuiu o tamanho dos frutos, mas não influenciou a qualidade do fruto avaliada pela porcentagem de suco, pelos sólidos solúveis totais, pela acidez total e pela relação sólidos/acidez. Foram observados efeitos positivos do P na produção, na porcentagem de suco e na relação sólidos/ acidez. O K aumentou a produção, o tamanho dos frutos e a acidez, mas diminuiu a relação sólidos / acidez.
\end{abstract}

Termos para indexação: laranja, fertilização, macronutrientes.

\author{
RESPONSE OF ORANGE TREES TO NITROGEN, PHOSPHORUS AND POTASSIUM \\ FERTILIZATION IN A YELLOW LATOSOL OF THE COASTAL PLATEAU
}

\begin{abstract}
The effects of N, P, and K applications on yield and fruit quality of orange (Citrus sinensis (L.) Osbeck) was studied in a factorial experiment carried out in a Yellow Latosol, of the Coastal Plateau of the Northeast of Brazil. Zero, 150 e $300 \mathrm{~kg} \mathrm{ha}^{-1} \mathrm{~N}$ as urea; 0,26 and $52 \mathrm{~kg} \mathrm{ha}^{-1}$ of P as concentrated superfosfate and 0,96 and $192 \mathrm{~kg} \mathrm{ha}^{-1}$ of $\mathrm{K}$ as $\mathrm{KCl}$ were used. A linear yield response to $\mathrm{N}$ was observed in one, out of four years of observation. Nitrogen decreased fruit size and had no influence on fruit quality measured by percentage of juice, total soluble solids, acidity and ratio total soluble solids/acidity. Yield was highly responsive to $\mathrm{P}$ in each year observed. The nutrient increased percentage of juice and the total soluble solids/acidity ratio. Potassium increased yield and fruit size, but decreased the total soluble solids/acidity ratio.
\end{abstract}

Index terms: orange, fertilization, macronutrients.

\footnotetext{
${ }^{1}$ Aceito para publicação em 26 de julho de 1999.

${ }^{2}$ Eng. Agrôn., Ph.D., Embrapa-Centro de Pesquisa Agropecuária dos Tabuleiros Costeiros (CPATC), Caixa Postal 44, CEP 49025-040 Aracaju, SE. E-mail: lafayete@cpatc.embrapa.br

${ }^{3}$ Eng. Agrôn., M.Sc., Embrapa-Centro Nacional de Pesquisa de Mandioca e Fruticultura (CNPMF), Caixa Postal 007, CEP 44380-000 Cruz das Almas, BA.

${ }^{4}$ Eng. Agrôn., M.Sc., Empresa de Desenvolvimento Agropecuário de Sergipe (Emdagro), Centro Administrativo Augusto Franco, Caixa Postal 297, CEP 49080-190 Aracaju, SE

${ }^{5}$ Eng. Agrôn., M.Sc., Embrapa-CPATC.
}

\section{INTRODUÇÃO}

No Nordeste do Brasil, a citricultura está instalada em solos dos Tabuleiros Costeiros, onde predominam os Latossolos e os Podzólicos, ambos originários do terciário, com alto grau de intemperização (Jacomine, 1996). Cada tonelada de laranja produzida exporta 2,080 kg de N, 0,185 kg de P e 1,505 kg de K (Malavolta, 1983). Embora as recomendações de fertilizantes não possam se basear somente nos nutrientes exportados, pois quando estão no solo volatilizam-se, 
lixiviam-se e reagem com os constituintes do solo, diminuindo assim a disponibilidade para as plantas; esses números dão uma indicação das quantidades a serem repostas.

Quaggio (1992) observou respostas significativas positivas da laranja à aplicação de $\mathrm{N}$, tanto no Podzólico Vermelho-Amarelo eutrófico quanto no Latossolo Vermelho-Escuro eutrófico. A dose calculada para máxima produtividade nos dois solos foi de $220 \mathrm{~kg} \mathrm{ha}^{-1}$.

Apesar de as quantidades de $\mathrm{P}$ requeridas pela planta cítrica serem bem menores, quando comparadas com as de Ca, N e K (Davies \& Albrigo, 1994), em solos tropicais, onde o teor de $\mathrm{P}$ no solo é muito baixo, ele age como fator limitante da produção. Quaggio (1992) observou resposta linear ao P, até a dose de $140 \mathrm{~kg} \mathrm{ha}^{-1}$ de $\mathrm{P}$, quando o teor do nutriente no solo era $4 \mathrm{mg} \mathrm{dm}^{-3}$, pelo método da resina trocadora de íons. Nos solos onde os teores de $\mathrm{P}$ eram maiores que $9 \mathrm{mg} \mathrm{dm}^{-3}$ pelo mesmo método, não foram observadas respostas.

A adubação potássica é importante, particularmente quando o objetivo é produzir para o mercado in natura, pois sua deficiência não somente causa queda de frutos, mas também impede o crescimento dos que permanecem. Quaggio (1992) encontrou respostas lineares ao $\mathrm{K}$ quando o teor do nutriente em um LE distrófico era $27,3 \mathrm{mg} \mathrm{dm}^{-3}$. Em relação à qualidade de fruto, Du Plessis \& Koen $(1984,1989)$ observaram aumento do tamanho do fruto com aplicação de K.

O objetivo deste trabalho foi estudar o efeito do $\mathrm{N}, \mathrm{P}$ e K na produção e qualidade dos frutos da laranja-pêra, em um Latossolo Amarelo dos Tabuleiros Costeiros.

\section{MATERIAL E MÉTODOS}

Foi instalado um experimento fatorial $3^{3}$, com três blocos incompletos com confundimento $\mathrm{W}$ em um Latossolo Amarelo dos Tabuleiros Costeiros, com as seguintes características: $\mathrm{pH}$ em água $(1: 2,5) 5,5$; C.O. (oxidação pelo dicromato de $\mathrm{K}$ a quente) $11,6 \mathrm{~g} \mathrm{~kg}^{-1}$; $\mathrm{Ca}^{2+}+\mathrm{Mg}^{2+}$ (extração com $\mathrm{KCl} \mathrm{N}$ e titulação com EDTA) $22 \mathrm{mmol}_{\mathrm{c}} \mathrm{kg}^{-1} ; \mathrm{Al}^{3+}$ ( extração com $\mathrm{KCl} \mathrm{N}$ e titulação com $\mathrm{NaOH}) 1 \mathrm{mmol}_{\mathrm{c}} \mathrm{kg}^{-1}$; P(Mehlich 1) $1,1 \mathrm{mg} \mathrm{kg}^{-1}$; $\mathrm{K}$ (Mehlich 1) $47 \mathrm{mg} \mathrm{kg}^{-1}$; areia $846 \mathrm{~g} \mathrm{~kg}^{-1}$; silte $81 \mathrm{~g} \mathrm{~kg}^{-1}$ e argila $73 \mathrm{~g} \mathrm{~kg}^{-1}$, cujas determinações foram efetuadas de acordo com Embrapa (1979). Neste experimento, usou-se a laranja-pêra clone D6 sobre limão-cravo. O espaçamento utilizado foi de $6 \mathrm{~m}$ x $6 \mathrm{~m}$. Foi utilizada parcela útil de $216 \mathrm{~m}^{2}$ constituída de seis plantas. Uma linha de plantas foi utilizada como bordadura para separar uma parcela da outra. O plantio foi efetuado em 1973. As doses de N, P e K foram, respectivamente, 0 , 150 e $300 \mathrm{~kg} \mathrm{ha}^{-1}$ de $\mathrm{N}$ na forma de uréia, 0,26 e $52 \mathrm{~kg} \mathrm{ha}^{-1}$ de P na forma de superfosfato triplo, e 0,96 e $192 \mathrm{~kg} \mathrm{ha}^{-1}$ de $\mathrm{K}$ na forma de cloreto de K. Do primeiro ao sexto ano, as doses foram aplicadas de acordo com a fração $1 / 6$ até chegar-se a $6 / 6$ da mesma. $\mathrm{O} \mathrm{N}$ e o $\mathrm{K}$ foram fracionados em duas aplicações nos meses março/abril e julho/agosto quando ocorrem as duas principais floradas. Todos os fertilizantes foram aplicados em uma faixa entre a projeção da copa e a entrelinha.

Amostras de solo foram coletadas por tratamento, na projeção da copa das plantas úteis, na profundidade 0-20 cm. Durante a condução do experimento, foram efetuadas calagens, visando insolubilizar o $\mathrm{Al}^{3+}$ ou elevar os teores de $\left(\mathrm{Ca}^{2+}+\mathrm{Mg}^{2+}\right)$ para $20 \mathrm{mmol}_{\mathrm{c}} \mathrm{kg}^{-1}$ de solo. Foram anotados o número e o peso dos frutos e calculado o peso médio de cada fruto. Neste trabalho, foram incluídos os anos de 1979, 1981, 1984 e 1985. Amostras de frutos das plantas úteis de cada tratamento foram coletadas nos anos de 1981 e 1985, nas quais foi determinada a porcentagem de suco, de sólidos solúveis totais, de acidez total, e calculada a relação sólidos solúveis totais/acidez (Salibe, 1974 ).

Os dados foram submetidos às análises estatísticas de variância e de regressão, de acordo com Snedecor \& Cochran (1980), e os graus de liberdade da interação tripla foram utilizados para testar os efeitos dos tratamentos.

\section{RESULTADOS E DISCUSSÃO}

O solo onde foi conduzido o experimento não dispõe de minerais primários, e não pode repor os nutrientes removidos pela colheita (Sobral, 1984). Os teores de matéria orgânica são baixos, e os valores de $\mathrm{pH}$ e teores de $\mathrm{Ca}^{2+}+\mathrm{Mg}^{2+}$ e $\mathrm{Al}^{3+}$ estão de acordo com o critério de cálculo de calagem utilizado durante a condução do trabalho (Tabela 1).

$\mathrm{O}$ efeito do $\mathrm{N}$ e da interação $\mathrm{N}$ x P foi significativo em apenas um dos quatro anos considerados. A fonte utilizada foi a uréia, cuja volatilização pode aumentar se na época da aplicação as condições de clima (temperatura) e solo (umidade) forem desfavoráveis, além da lixiviação, a qual foi favorecida pelo baixo 
teor de argila do solo onde foi conduzido o experimento. A falta de consistência na resposta ao $\mathrm{N}$ pode ser atribuída ao fato de que as plantas cítricas armazenam $\mathrm{N}$ principalmente nas folhas e nas partes lenhosas da planta (tronco e ramos), utilizando-o na época da brotação ( Legaz et al., 1982). Dou et al. (1997) estudaram a contribuição em N proveniente da mineralização de resíduos como folhas e galhos secos que retornam ao solo, e concluíram que esses resíduos podem contribuir com até $153 \mathrm{~kg} \mathrm{ha}^{-1} \mathrm{ano}^{-1}$ quantidade significativa em relação ao requerimento anual de $\mathrm{N}$ pela planta cítrica. $\mathrm{O} \mathrm{N}$ diminuiu significativamente o peso médio do fruto nos anos de 1981 e 1985, e não influenciou significativamente o teor de sólidos totais, acidez e relação sólidos acidez. Tais resultados estão de acordo com os encontrados por Silva et al. (1984), os quais não encontraram efeito do nutriente nas referidas variáveis.

Embora as plantas cítricas necessitem de $\mathrm{P}$ em quantidades bem menores do que as quantidades de N e K (Davies \& Albrigo, 1994), o P pode limitar drasticamente a produção, quando o seu teor no solo for muito baixo (Quaggio, 1992 ). Na Fig. 1, é mostrado o efeito do $\mathrm{P}$ sobre a produção de frutos, considerando a média dos quatro anos. Foram observados efeitos significativos do $\mathrm{P}$ na produção de frutos em todos os anos. A produção máxima foi obtida com a aplicação de $41,5 \mathrm{~kg} \mathrm{ha}^{-1}$ de $P$. Considerando o preço do $\mathrm{P}$ de $\mathrm{R} \$ 2,29 \mathrm{~kg}^{-1}$ e o preço da laranja $\mathrm{R} \$ 50,00 \mathrm{t}^{-1}$, a dose de $\mathrm{P}$ que propicia $\mathrm{o}$ máximo lucro líquido é a de $39,52 \mathrm{~kg} \mathrm{ha}^{-1} \mathrm{de} \mathrm{P}$. As aplicações anuais de $\mathrm{P}$ fizeram com que o nutriente se acumulasse no solo (Fig. 2). O P aumentou significativamente o peso médio dos frutos, a porcentagem de suco e a relação sólidos solúveis/acidez, diminuindo também significativamente a acidez e os sólidos solúveis (Figs. 3 e 4). Os resultados encontrados estão de acordo com Malavolta (1983) exceto em relação à razão sólidos/acidez.

Na Fig. 5, é mostrado o efeito do K na produção média de frutos nos últimos dois anos, cujas respostas foram quadráticas. $\mathrm{O} \mathrm{K}$ aumentou significativamente a produção de frutos em três dos quatro anos considerados. A produção máxima foi obtida com a aplicação de $150 \mathrm{~kg} \mathrm{ha}^{-1}$ de K. Considerando o preço do $\mathrm{K}$ de $\mathrm{R} \$ 0,504 \mathrm{~kg}^{-1}$ e o preço da laranja $\mathrm{R} \$ 50,00 \mathrm{t}^{-1}$, a dose de $\mathrm{K}$ que propicia o máximo lucro líquido é de $138,94 \mathrm{~kg} \mathrm{ha}^{-1}$ de $\mathrm{K}$. As aplicações anuais de $\mathrm{K}$ fizeram com que o nutriente também se acumulasse no solo (Fig. 6). O nutriente aumentou o peso médio dos frutos, o qual diminuiu com a idade da planta, independentemente da dose de K (Fig. 7). Esses resultados estão de acordo com os encontrados por Quaggio (1992), em relação à produção, e por Du Plessis \& Koen (1984,1989), em relação ao tamanho dos frutos. A acidez foi elevada, e a relação sólidos solúveis/acidez reduzida significativamente pelo K (Fig. 8). O K causou diminuição dos sólidos solúveis, embora o efeito não tenha sido significativo.

TABELA 1. Matéria orgânica, $\mathrm{pH}, \mathrm{Ca}+\mathrm{Mg}$ e $\mathrm{Al}$ no solo, em duas épocas de amostragem, efetuadas na projeção da copa, na profundidade $d e-20 \mathrm{~cm}$.

\begin{tabular}{|c|c|c|c|c|c|c|c|c|}
\hline \multirow[t]{2}{*}{ Tratamento } & \multicolumn{4}{|c|}{ Três anos após o plantio } & \multicolumn{4}{|c|}{ Dez anos após o plantio } \\
\hline & M.O. & $\mathrm{pH}$ & $(\mathrm{Ca}+\mathrm{Mg})$ & $\mathrm{Al}$ & M.O. & $\mathrm{pH}$ & $(\mathrm{Ca}+\mathrm{Mg})$ & $\mathrm{Al}$ \\
\hline & $\left(\mathrm{g} \mathrm{kg}^{-1}\right)$ & & $----(\mathrm{mmc}$ & 1)--- & $\left(\mathrm{g} \mathrm{kg}^{-1}\right)$ & & $----(\mathrm{mm}$ & )- \\
\hline $\mathrm{N} 0$ & 20,3 & 5,6 & 21,1 & 0,6 & 12,0 & 5,7 & 14,9 & 0,1 \\
\hline N 1 & 19,9 & 5,1 & 21,2 & 1,8 & 12,8 & 5,0 & 15,1 & 0,1 \\
\hline N 2 & 19,6 & 4,8 & 21,2 & 2,1 & 12,9 & 4,8 & 16,8 & 0,2 \\
\hline P 0 & 19,3 & 4,9 & 20,6 & 2,2 & 10,8 & 5,1 & 7,6 & 0,2 \\
\hline P 1 & 19,1 & 5,2 & 19,1 & 1,4 & 13,1 & 5,2 & 16,9 & 0,1 \\
\hline P 2 & 21,3 & 5,4 & 23,9 & 0,8 & 13,8 & 5,3 & 22,3 & 0,1 \\
\hline K 0 & 19,7 & 5,1 & 22,0 & 1,6 & 12,2 & 5,2 & 17,9 & 0,1 \\
\hline K 1 & 19,9 & 5,2 & 22,4 & 1,3 & 12,9 & 5,2 & 14,6 & 0,1 \\
\hline K 2 & 20,2 & 5,3 & 19,1 & 1,6 & 12,6 & 5,2 & 14,3 & 0,1 \\
\hline
\end{tabular}




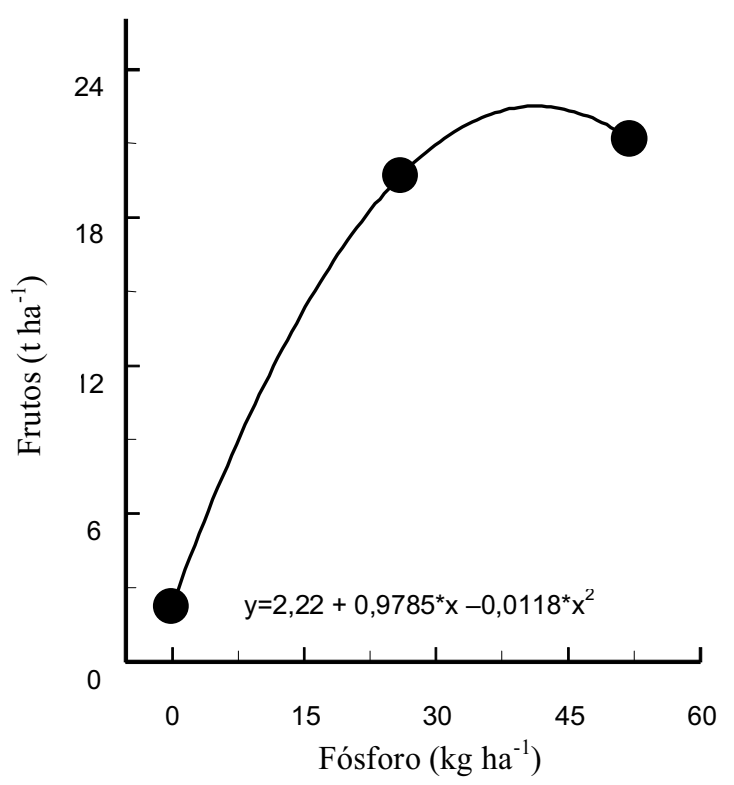

FIG. 1. Efeitos do P (média de quatro anos) na produção de frutos.

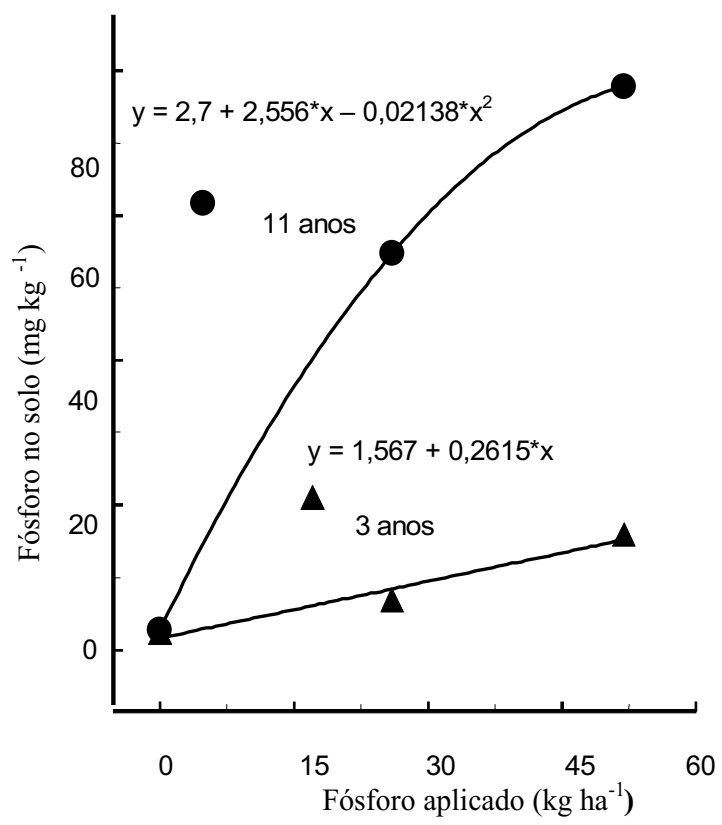

FIG. 2. Acúmulo de $P$ no solo. Amostra coletada na profundidade de $0-20 \mathrm{~cm}$.

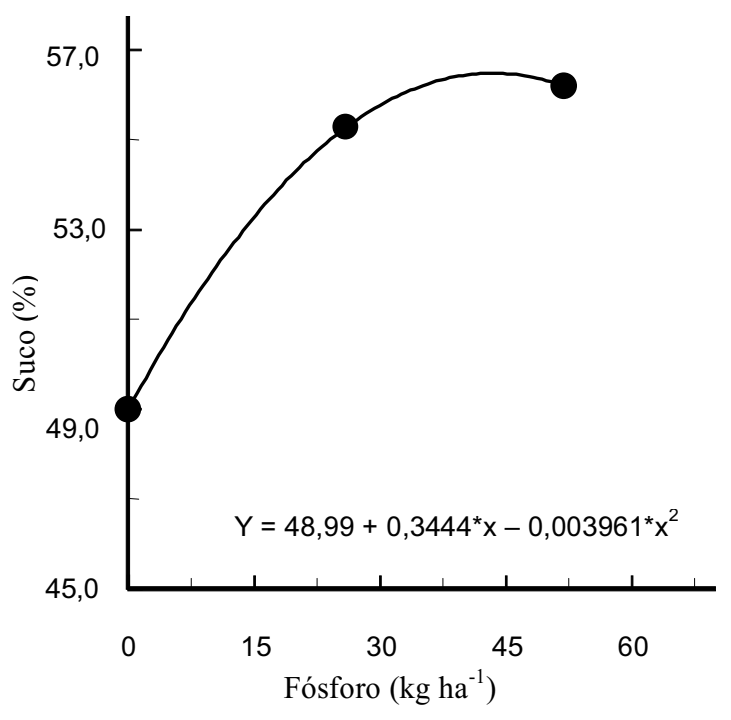

FIG.3. Efeito do $P$ na porcentagem de suco da laranja-pêra, 1981.

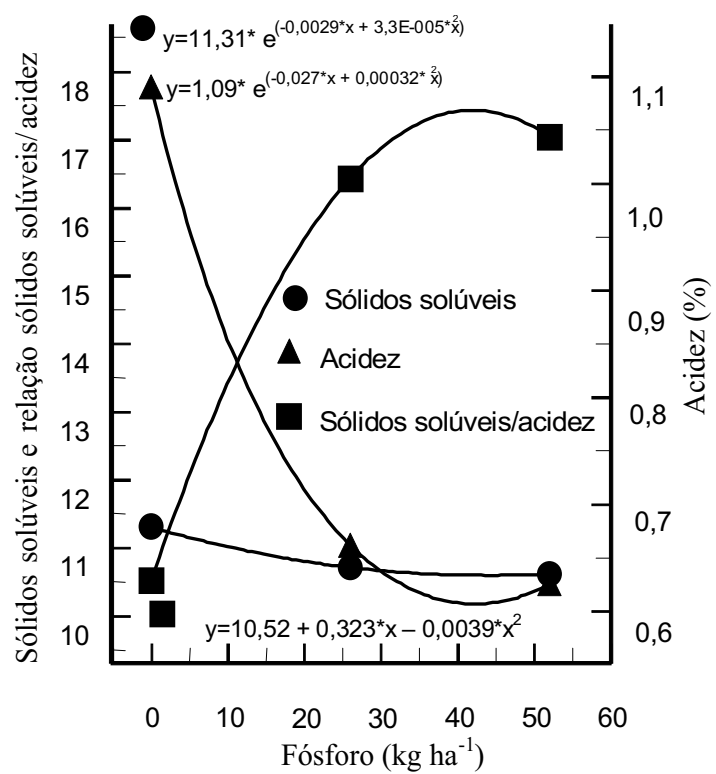

FIG. 4. Efeitos do $P$ na acidez, sólidos solúveis e relação sólidos solúveis/acidez da laranja-pêra, 1981. 


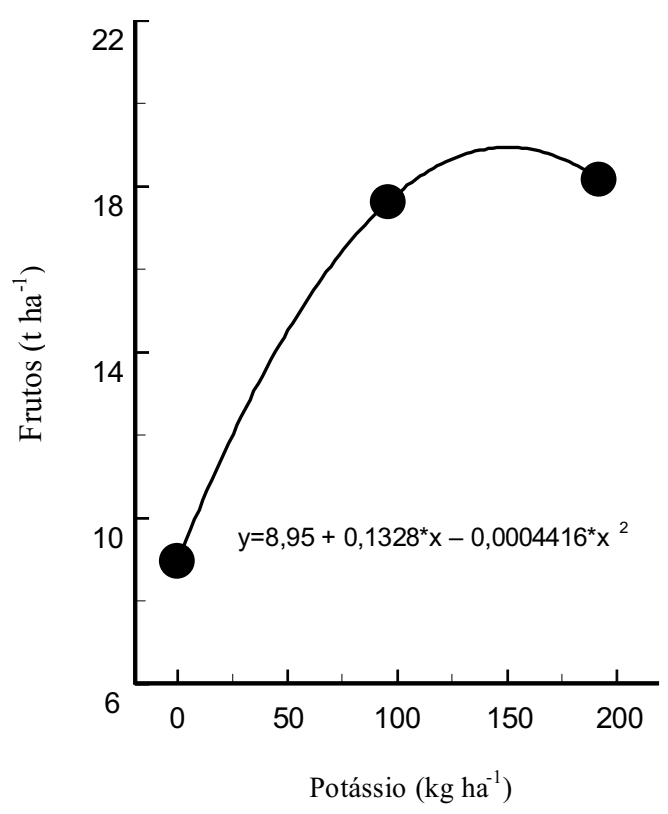

FIG. 5. Efeitos do K (média de dois anos) na produção de frutos de laranja-pêra.

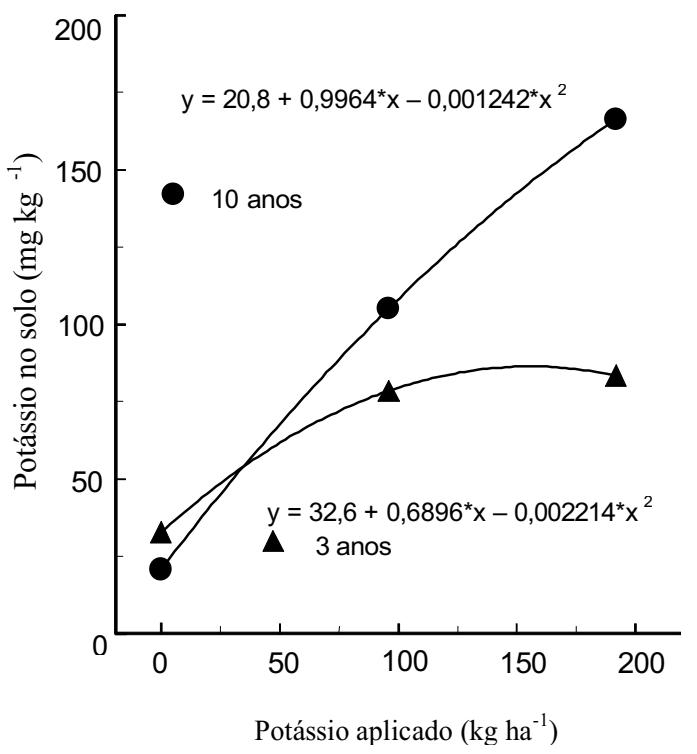

FIG. 6. Acúmulo de K no solo. Amostra coletada na profundidade de $0-20 \mathrm{~cm}$.

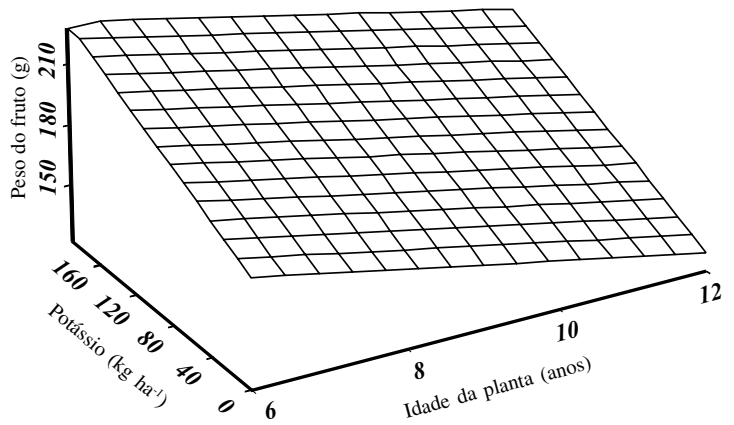

FIG. 7. Influência do $\mathrm{K}$ e da idade da planta no peso do fruto da laranjeira-pêra.

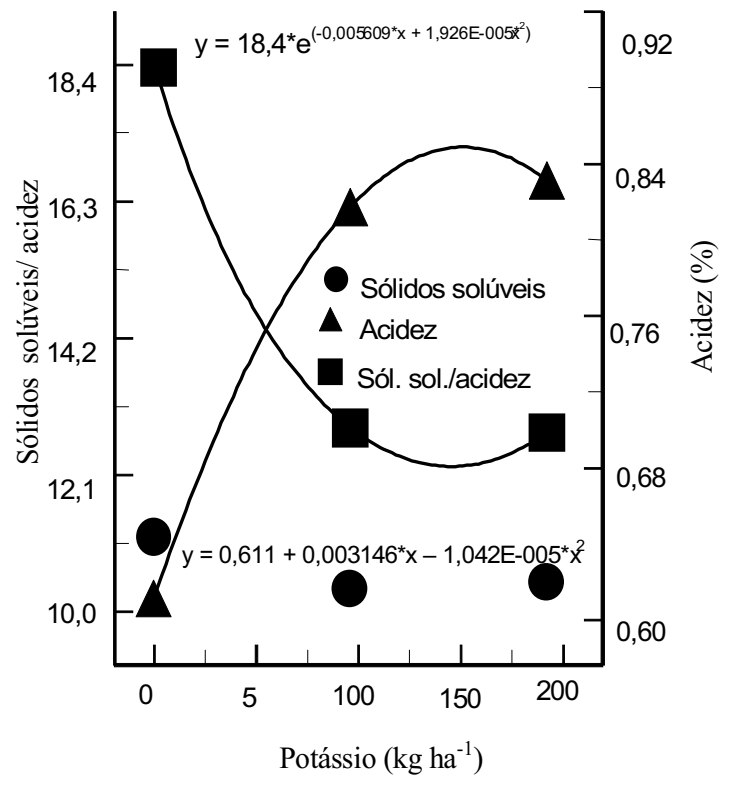

FIG. 8. Efeito do $K$ na acidez, sólidos solúveis e relação sólidos solúveis/acidez da laranja-pêra, 1985.

\section{CONCLUSÕES}

1. O P e o K aumentam a produção.

2. $\mathrm{O} \mathrm{N}$ diminui o peso médio dos frutos e não influi na qualidade deles.

3. O P aumenta a porcentagem de suco e o K, o tamanho dos frutos; a relação sólidos solúveis/acidez aumenta com a aplicação de $\mathrm{P}$, e diminui com a de $\mathrm{K}$. 


\section{REFERÊNCIAS}

DAVIES, F.S.; ALBRIGO, L.G. Citrus. Wallingford, UK : CAB International, 1994. 254p.

DOU, H.; ALVA, A.K.; KHAKURAL, B.R. Nitrogen mineralization from citrus trees residues under different production conditions. Soil Science Society of America. Journal, Madison, v.61, p.1226-1232, 1997.

DU PLESSIS, S.F.; KOEN, T.J. Effect of nutrition on fruit size of citrus. International Society of Citriculture Proceedings, Riverside, v.1, p.148-154, 1984.

DU PLESSIS S.F.; KOEN, T.J. The effect of $\mathrm{N}$ and $\mathrm{K}$ fertilization on yield and fruit size of valencia orange. Potash Review, Worblaufen-Borne, n.1, p.1-7, 1989.

EMBRAPA. Serviço Nacional de Levantamento e Conservação de Solos (Rio de Janeiro, RJ). Manual de análise de solo. Rio de Janeiro, 1979. Não paginado.

JACOMINE, P.K.T. Distribuição geográfica, características e classificação dos solos coesos dos Tabuleiros Costeiros. In: REUNIÃO TÉCNICA SOBRE SOLOS COESOS DOS TABULEIROS COSTEIROS, 1996, Cruz das Almas. Aracaju : EmbrapaCPATC-CNPMF/EAUFBA/IGUFBA, 1996. p.1326.

LEGAZ, F.; PRIMO-MILLO, E.; PRIMO YUFERA, E.; GIL, C.; RUBIO, J.L. Nitrogen fertilization in citrus.
1. Absorption and distribution in calamondin trees (Citrus mitis. BI.), during flowering, fruit set and initial fruit development periods. Plant and Soil, Dordrecht, v.66, n.3, p.339-351, 1982.

MALAVOLTA, E. Nutrição mineral e adubação da laranjeira. In: YAMADA,T. (Ed.). Nutrição mineral e adubação dos citros. Piracicaba : Potafós, 1983. p.13-68.

QUAGGIO, J.A. Conceitos modernos sobre calagem e adubação para citros no Estado de São Paulo. Laranja, v.13, n.27, p.457-488, 1992.

SALIBE, A.A. Curso de especialização em fruticultura: cultura dos citros. 3.ed. Recife : SUDENE/ UFRPe, 1974. 188p.

SILVA, J.U.B.; SOBRAL, L.F.; FONSECA, A.J.de; TRINDADE, J.; SILVA, L.M.S. Effect of different sources and dosage-splitting of nitrogen upon the growth yield and fruit quality of "Baianinha" orange. Proceedings of the International Society of Citriculture, v.1, p.154-156, 1984.

SNEDECOR, G.W.; COCHRAN,W.G. Statistical methods. 7.ed. Ames, Iowa : The Iowa State University Press, 1980. 507p.

SOBRAL, L.F. Phosphorus availability as influenced by chemical and mineralogical properties of Sergipe State Soils, Brazil. College Station, Texas : Texas A \& M University, 1984. 61p. Ph.D. Thesis. 